\title{
CORRECTION
}

\section{Correction to: Non-response in surveys of very old people}

\author{
Michael Wagner ${ }^{1} \cdot$ Matthias Kuppler $^{1} \cdot$ Christian Rietz $^{2} \cdot$ Roman Kaspar $^{3}$
}

Published online: 15 October 2018

(c) Springer Nature B.V. 2018

\section{Correction to: European Journal of Ageing https://doi.org/10.1007/s10433-018-0488-x}

In the original publication of the article, Table 3 was aligned incorrectly. Now the same has been formatted in this correction.

The original article has been corrected and can be found online at https://doi.org/10.1007/s10433-018-0488-x.

Michael Wagner

mwagner@wiso.uni-koeln.de

Matthias Kuppler

kuppler@wiso.uni-koeln.de

Christian Rietz

christian.rietz@ph-heidelberg.de

Roman Kaspar

roman.kaspar@uni-koeln.de

1 Institute of Sociology and Social Psychology, University of Cologne, Cologne, Germany

2 Institute for Educational Science, Heidelberg University of Education, Heidelberg, Germany

3 Ceres - Cologne Center for Ethics, Rights, Economics, and Social Sciences of Health, University of Cologne, Cologne, Germany 
Table 3 Logistic regression of contact, cooperation, response, and refusal rate on type of residence, age, and sex (base model, weighted)

\begin{tabular}{|c|c|c|c|c|c|c|c|c|c|c|c|c|}
\hline \multirow{3}{*}{$\begin{array}{l}\text { Variable } \\
\text { Type of residence } \\
\text { (ref.: institutional setting) }\end{array}$} & \multicolumn{3}{|l|}{ Contact } & \multicolumn{3}{|l|}{ Cooperation } & \multicolumn{3}{|l|}{ Response } & \multicolumn{3}{|l|}{ Refusal } \\
\hline & \multirow{2}{*}{$\begin{array}{l}\mathrm{OR} \\
1.2\end{array}$} & \multicolumn{2}{|c|}{$95 \% \mathrm{CI}$} & \multirow{2}{*}{$\begin{array}{l}\mathrm{OR} \\
1.04\end{array}$} & \multicolumn{2}{|c|}{$95 \% \mathrm{CI}$} & \multirow{2}{*}{$\frac{\overline{\mathrm{OR}}}{1.22}$} & \multicolumn{2}{|c|}{$95 \% \mathrm{CI}$} & \multirow{2}{*}{$\begin{array}{l}\mathrm{OR} \\
2.3\end{array}$} & \multicolumn{2}{|c|}{$95 \% \mathrm{CI}$} \\
\hline & & 0.84 & 1.71 & & 0.72 & 1.49 & & 0.88 & 1.71 & & 1.58 & 3.34 \\
\hline \multicolumn{13}{|l|}{ Age-group (ref. 90+) } \\
\hline $80-84$ & 1.07 & 0.74 & 1.55 & 0.77 & 0.54 & 1.11 & 0.82 & 0.59 & 1.14 & 1.84 & 1.29 & 2.62 \\
\hline $85-89$ & 1.36 & 0.94 & 1.96 & 0.93 & 0.67 & 1.3 & 1.02 & 0.75 & 1.39 & 1.73 & 1.24 & 2.42 \\
\hline Sex (ref. male) & 1.22 & 0.89 & 1.69 & 0.85 & 0.63 & 1.13 & 0.91 & 0.7 & 1.2 & 1.45 & 1.10 & 1.92 \\
\hline Constant & 1.62 & 0.9 & 2.92 & 1.31 & 0.73 & 2.35 & 0.55 & 0.32 & 0.96 & 0.06 & 0.03 & 0.11 \\
\hline$N$ & 1505 & & & 1176 & & & 1505 & & & 1505 & & \\
\hline$F(d f$, design $d f)$ & $5.93(9,1496)$ & & & $2.93(9,1167)$ & & & $3.27(9,1496)$ & & & $6.31(9,1496)$ & & \\
\hline Prob $>F$ & $<0.001$ & & & 0.002 & & & $<0.001$ & & & $<0.001$ & & \\
\hline
\end{tabular}

Models control for sampling points

OR, odds ratio; 95\% CI, 95\% confidence interval; $F(d f$, design $d f)=F$ test of overall model fit; Prob $>F=p$ value of the $F$ test 